OPEN ACCESS

Edited by:

Kelly Russell,

University of Manitoba, Canada

Reviewed by:

Meeryo Christa Choe,

UCLA Mattel Children's Hospital,

United States

Michael Cusimano

St Michael's Hospital, Canada

*Correspondence:

Alice Theadom

alice.theadom@aut.ac.nz

Specialty section:

This article was submitted to

Neurorehabilitation,

a section of the journal

Frontiers in Neurology

Received: 10 August 2021 Accepted: 09 December 2021

Published: 05 January 2022

Citation:

Brady M, Hume PA, Mahon S and

Theadom A (2022) What is the

Evidence on Natural Recovery Over the Year Following Sports-Related and

Non-sports-Related Mild Traumatic

Brain Injury: A Scoping Review.

Front. Neurol. 12:756700.

doi: 10.3389/fneur.2021.756700

\section{What Is the Evidence on Natural Recovery Over the Year Following Sports-Related and Non-sports-Related Mild Traumatic Brain Injury: A Scoping Review}

\author{
Morgan Brady ${ }^{1}$, Patria A. Hume ${ }^{1,2}$, Susan Mahon ${ }^{1}$ and Alice Theadom ${ }^{1 *}$ \\ ${ }^{1}$ Traumatic Brain Injury Network (TBIN), Faculty of Health and Environmental Sciences, Auckland University of Technology, \\ Auckland, New Zealand, ${ }^{2}$ Sport Performance Research Institute New Zealand (SPRINZ), Faculty of Health and Environmental \\ Sciences, Auckland University of Technology, Auckland, New Zealand
}

Background: Treatment approaches often differ dependent upon whether a person experiences a sports-related or a non-sports-related mild traumatic brain injury. It remains unclear if recovery from these injuries is comparable or unique to context of the injury.

Objective: To identify knowledge gaps on self-reported outcomes and trajectories between sports- and non-sports-related mild traumatic brain injuries and how they are assessed in adults.

Methods: This scoping review used a systematic search of key electronic databases, including PubMed, SPORTDiscus, Embase, MEDLINE, and CINAHL for articles published in 1937 until March 10, 2021. Articles were included if they were available in English; full text published in a peer-reviewed journal; had a prospective or retrospective study design; reported data on mild TBI cases $>16$ years of age, and included data from at least two time points on self-reported outcomes within 12 months post-injury. A standardized data extraction spreadsheet was used to determine the participant characteristics, definitions, assessment methods, outcomes, and recovery time frames.

Results: Following removal of duplicates, the search strategy elicited 6,974 abstracts. Following abstract review, 174 were retained for full text review. Of the 42 articles that met inclusion criteria, 18 were sports related (15 in the USA and three in Canada) and 24 were general population studies (six in USA, three in Canada, three in Australasia, nine in Europe, two in Taiwan, and one in Morocco). Direct comparison in recovery trajectories between the sport and general population studies was difficult, given notable differences in methodology, definitions, types of outcome measures, and timing of follow-up assessments. Only one article reported on both sports-related and non-sportsrelated traumatic brain injuries separately at comparable timepoints. This study revealed no differences in recovery time frames or overall symptom burden. 
Discussion: Whilst there is a clear benefit in researching specific subpopulations in detail, standardized outcome measures and follow-up time frames are needed across contexts to facilitate understanding of similarities and differences between sports- and non-sports-related mild traumatic brain injuries to inform clinical treatment.

Keywords: traumatic brain injury, concussion, review, recovery, sport

\section{INTRODUCTION}

A mild traumatic brain injury is defined as a traumatically induced transient disturbance of brain function (1). A concussion is often considered to be a subset of mTBI (2). There is considerable debate as to whether concussion is a useful term, with some advocating the term has no clear definition (3), no pathological meaning (4), and lacks diagnostic precision $(4,5)$. However, others have argued that the term describes a distinct pathophysiological entity with its own diagnostic and management implications (4). Throughout this paper, only the terms mTBI or sports-related brain injury (SRTBI) $(2,6)$ have been used. Both these terms include cases of concussion.

Internationally, the reported age-adjusted incidence rates for the general population for mTBI vary between 59.6 and 811 per 100,000 (7), with approximately $80 \%$ of injuries classified as being of mild severity (8). Key challenges in the identification and management of mTBI are that patients can present to a range of services and health care professions (9). Even within countries, service provision can be highly variable (10).

Early recovery advice and gradual return to activity have been found to facilitate recovery (11). In many cases, people recover well in the days to weeks following injury; however, more recently, studies have shown that half of people experiencing mTBI report functional limitations (12) and persistent symptoms, including headaches, fatigue, forgetfulness, poor concentration, and impaired information processing (13). For those with persistent symptoms, interdisciplinary rehabilitation approaches have been found to effectively improve recovery (14).

Of the services providing interdisciplinary rehabilitation interventions, some services specifically focus on management of sports-related injuries, whereas other services treat all clinical presentations. Sports- and non-sports-related kinds of mTBI (e.g., injuries sustained during everyday activities, such as through falls, assaults, vehicle crashes or being hit by or hitting the head against an object) are often talked about, researched, and treated differently. Whilst context-specific research and rehabilitation are important to ensure issues such as managing motivation to return to sport too soon are explored, there is also a need to look across different contexts to advance the field as a whole. For example, what is the best treatment approach for an elite athlete who sustains an mTBI through a vehicle accident, is experiencing psychological trauma, but whose main goal is to return to sport, or the person who sustains a sports-related injury but whose main rehabilitation goal is to return to work and be able to drive?
Some studies have proposed that there are notable differences on the impact of the injury with significantly fewer sportsrelated injuries showing positive MRI findings in comparison to non-sports-related injuries (15). However, it currently remains unclear if there are differences in the subjective experience of recovery. To provide optimal treatment, it will be important for clinicians to be aware of and address any specific differences between injury contexts.

Existing systematic reviews have explored the experience of prolonged symptoms after mTBI; however, they have focused on particular contexts such as sports-related injuries (16) rather than exploring differences between contexts. Consequently, there is a gap in the existing literature exploring the current evidence based on subjective outcomes following sports-related and non-sportsrelated $\mathrm{mTBI}$.

\section{OBJECTIVE}

This scoping review aimed to identify knowledge gaps in selfreported outcomes and trajectories between sports- and nonsports-related kinds of mTBI and how they are assessed in adults.

\section{METHODS}

Scoping reviews are used to determine the scope of existing literature on a given topic, clarify key concepts or definitions, examine the way research has been conducted, identify key characteristics, and identify knowledge gaps. To ensure a robust approach to identification of the evidence, a systematic literature search is conducted. As scoping reviews aim to map the existing evidence rather than answer a discrete question (as in the case of a systematic review); formal critical appraisal tools are not incorporated into the approach (17). As the aim of this review was to determine what current research there was that identified self-reported outcomes and trajectories between sports and non-sports-related kinds of mTBI and how they are assessed in adults, we chose a scoping review approach rather than a systematic review. Therefore, the review was not registered with PROSPERO.

To identify relevant literature, a systematic search of key electronic databases was conducted from the start date of database entries until March 10, 2021. Databases included PubMed (1966 onward), SPORTDiscus (1985 onward), Embase (1947 onward), MEDLINE (1946 onward), and CINAHL (1937 onward). The search was conducted using Category 1 terms ("traumatic brain injury" OR "mild tbi" OR mtbi OR concuss* OR brain inj* OR head inj* OR "skull fracture" 
TABLE 1 | Search strategy.

\begin{tabular}{|c|c|}
\hline Search by & Title/abstract \\
\hline \multirow[t]{2}{*}{ Category 1 terms } & $\begin{array}{l}\text { "traumatic brain injury" OR "mild tbi" OR mtbi OR } \\
\text { concuss* OR brain inj” OR head inj }{ }^{\star} \text { OR "skull fracture" } \\
\text { OR "head trauma" OR "craniocerebral trauma" OR "head } \\
\text { impact" OR "craniocerebral injury" OR "brain trauma" }\end{array}$ \\
\hline & AND \\
\hline Category 2 terms & $\begin{array}{l}\text { "community participation" OR "physical symptom" OR } \\
\text { "cognitive symptom" OR "emotional symptom" OR } \\
\text { postconcuss* symptom OR post-concuss* symptom OR } \\
\text { "symptom improvement" OR mood OR emotion OR } \\
\text { "clinical outcome" OR "clinical recovery" OR reintegration }\end{array}$ \\
\hline
\end{tabular}

OR "head trauma" OR "craniocerebral trauma" OR "head impact" OR "craniocerebral injury" OR "brain trauma”) and Category 2 terms ("community participation" OR "physical symptom" OR "cognitive symptom" OR "emotional symptom" OR postconcuss* symptom OR post-concuss* symptom OR "symptom improvement" OR "mood" OR "emotion" OR "clinical outcome" OR "clinical recovery" OR "reintegration"). The use of the search terms "concuss*" and "brain inj*" captured the relevant MeSH terms, including "brain concussion," "postconcussion syndrome," and "brain injuries." An example search strategy is given in Table $\mathbf{1}$.

To establish some control over heterogeneity of different studies (3), inclusion criteria were established. Papers were included if they met inclusion criteria with the study: available in English; published in a peer-reviewed journal; had full text available; had prospective or retrospective study designs; reported data on participants who had suffered from a mild TBI separately; had participants aged over 16 years or age groups were separated; and included at least one follow-up assessment of self-reported symptoms, mood, cognitive or physical functioning, and quality of life/participation within 12 months post-TBI. As the review aimed to explore the evidence on the recovery experience for the affected person, objective measures such as biomarkers and brain imaging were not included. Additionally, as the aim was to identify natural symptom experience and recovery, to prevent skewing the findings, intervention studies (designed to improve the subjective experience) were excluded.

Articles with both children and adults were included if data were split by age to enable extraction of adult ( $>16$ years) data only. The same process was completed for severity of injury; articles which covered multiple injury severities were only included if mTBI data were able to be analyzed separately for outcome measures. Studies including biomarkers, imaging, or mTBI as an outcome, pharmacology, or rehabilitation were excluded. Studies exploring predictors or influences of outcomes were included if data from more than one time point were available. If multiple articles utilized the same data for analysis, only one was included to avoid duplication of results. Articles retrieved from the search were screened at the abstract level by one author (MB). Two reviewers then independently reviewed full-text articles against the inclusion criteria and resolved any difference in opinion through discussion. One author (MB) extracted the data. A third reviewer was available to resolve any disagreement in the selection process; however, this was not required. All eligible citations were extracted into EndNote (version X9.2). A data extraction form was created in Excel with column headings used to inform the data to be extracted. For the purposes of answering the research question, data were extracted on; first author, year of publication, country, study aim, if a preinjury evaluation was conducted, details of the control group (if included), definition of mTBI, number of mTBI participants, age of the participants, mechanisms of injury included, gender, types of outcomes studied, outcome assessments used, timing of follow-ups, most common symptoms reported, as well as data on recovery time frames.

\section{RESULT}

A total of 12,212 references published up to March 10, 2021 were identified through the initial search of CINAHL $(n=1,329)$, Embase $(n=4,960)$, MEDLINE $(n=4,235)$, Pubmed $(n=1,002)$, and SPORTDiscus $(n=686)$ databases. After removal of duplicates, there were 6,974 abstracts for review. Of these, 174 full-text articles were reviewed. There were 42 articles that met the inclusion criteria. The selection process and reasons for exclusion are outlined in Figure 1.

On review of the included study populations, there were 18 studies that specifically presented data for sports-related injuries only (these were classified as sports-related studies and are shown in Table 2). There were 24 studies that provided data across all contexts and mechanisms of injury, including sports-related injuries (classified as general population studies and shown in Table 3). Only one study (36) directly presented data, comparing sports- vs. non-sports-related injuries using the same assessments at the same time points.

The 42 studies meeting inclusion criteria underwent data extraction using a standardized form for information about participant demographics, number and timing of follow-ups sessions, testing methods, and outcomes measured. For the sports-related articles, information on the type of sport was included, while the mechanism of injury was included for nonsport articles (Tables 2, 3).

Description of the severity of injury was determined based on the reported Glasgow Coma Scale (GCS) score (58) in the nonsport injury-related articles, with a score of 13-15 being defined as a mild TBI. Three studies $(38,40,57)$ did not include the GCS score and described severity based on duration of posttraumatic amnesia $(<24 \mathrm{~h})$ and loss of consciousness $(<30 \mathrm{~min})$ immediately post-injury. Sport-related articles did not report the GCS score. Instead, severity was based on description of the injury by an athletic trainer or a sports physician. Nine studies also graded individuals based on the American Academy of Neurology or Concussion in Sport Group guidelines (18-21, 24, $26,27,29,34)$.

Of 18 sports-related mTBI studies included, only one (23) did not have participants who played football, and two $(31,33)$ did not include information about the type of sports played. Fifteen 


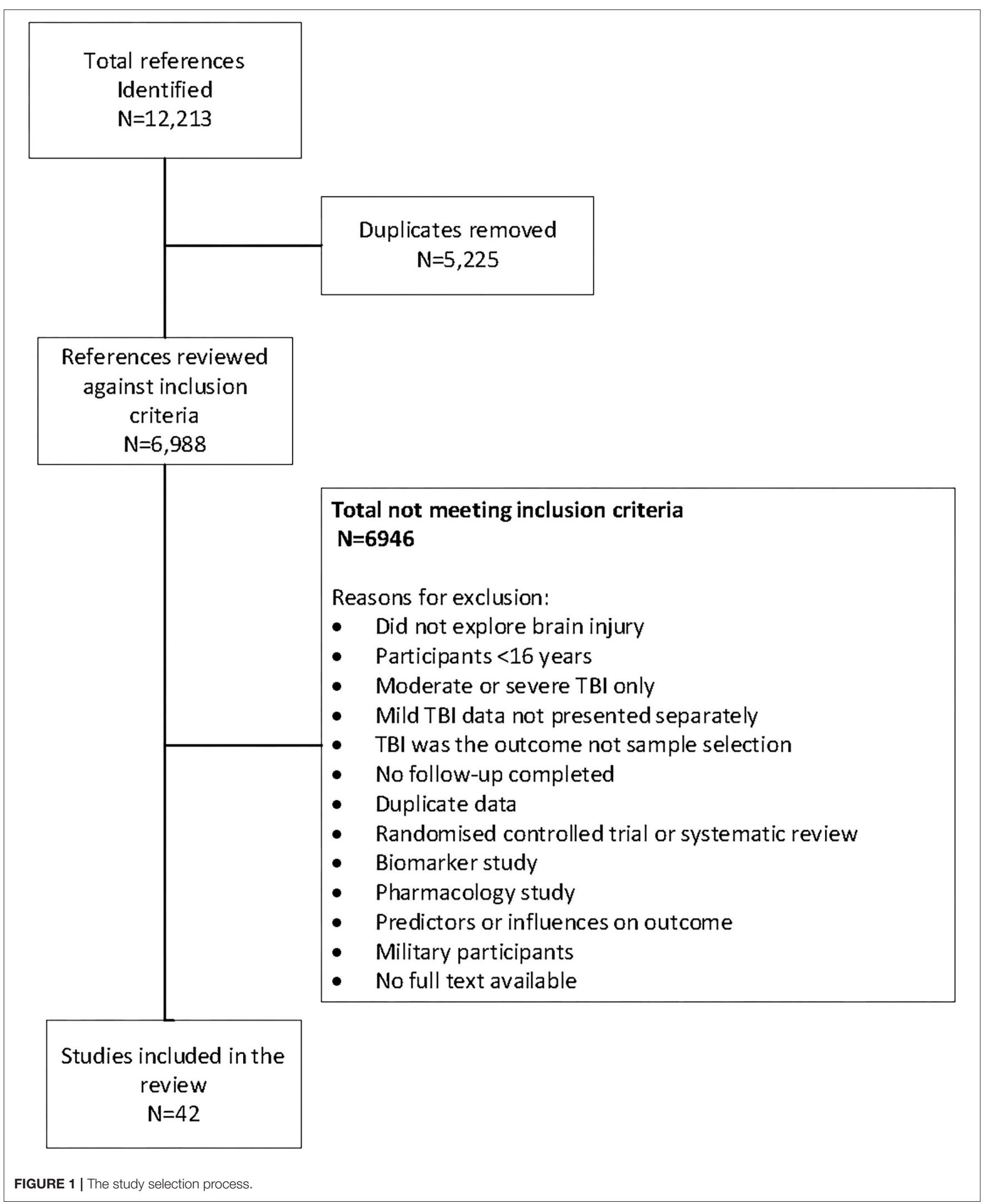


TABLE 2 | Sports-related studies.

\begin{tabular}{|c|c|c|c|c|c|}
\hline $\begin{array}{l}\text { First Author, } \\
\text { Year; } \\
\text { Country; } \\
\text { Reference }\end{array}$ & $\begin{array}{l}\text { Study sample } \\
\text { characteristics (age } \\
\text { range/ mean in } \\
\text { years); gender; sport }\end{array}$ & $\begin{array}{l}\text { Pre-injury Evaluation } \\
\text { (Y/N); Control group } \\
\text { included? (Y/N); } \\
\text { Number of } \mathrm{mTBI} \\
\text { participants; } \\
\text { Definition of } \mathrm{mTBI}\end{array}$ & Outcomes studied & $\begin{array}{l}\text { Tests used; Number of } \\
\text { follow-ups: mean (range); } \\
\text { Time of follow-up }\end{array}$ & $\begin{array}{l}\text { Mean outcome scores; Common } \\
\text { symptom presentation; Average } \\
\text { recovery time }\end{array}$ \\
\hline
\end{tabular}

\begin{tabular}{lll}
\hline $\begin{array}{l}\text { Black et al., } \\
\text { 2017; }\end{array}$ & $\begin{array}{l}\text { Varsity athletes; (20); } \\
\text { 44\% male; women's }\end{array}$ & $\begin{array}{l}\text { Y; Y; 75; Concussion in } \\
\text { Canada (18) }\end{array}$ \\
& $\begin{array}{l}\text { rugby, men's football, } \\
\text { women's hockey, men's } \\
\text { basketball, men's } \\
\text { hockey, field hockey. }\end{array}$ & $\begin{array}{l}\text { Diagnosed by student } \\
\text { therapist then sport } \\
\text { physician. }\end{array}$ \\
& $\begin{array}{l}\text { Collegiate athletes; } \\
\text { Collins et al., }\end{array}$ & Y; Y; 16; American \\
1999; USA & (20); 100\% male; & Academy of Neurology \\
(19) & football. & grading. Diagnosed by \\
& & $\begin{array}{l}\text { athletic trainer then } \\
\text { sport physician. }\end{array}$
\end{tabular}

Covassin et al., 2007; USA (20)

Covassin et al., 2012; USA (21)

Echemendia et al., 2001; USA (22)

Fait et al., 2013; Canada (23)
Collegiate athletes; (college age); 52\% male; wrestling, women's soccer, football, men's soccer, gymnastics, softball, lacrosse.

High school athletes; (19); mixed gender; football, women's soccer, men's soccer, women's volleyball, women's basketball, wrestling, men's basketball.

\section{Collegiate athletes;} (college age); mixed gender; football, men's soccer, men's ice hockey, women's basketball. basketball, men's
Y; N; 79; American Academy of Neurology grading. Diagnosed by athletic trainer then sport physician.

Y; N; 72; Concussion in Sport Group guidelines. Diagnosed by athletic trainer and sports physician.
Y; Y; 29; Participants described as having experienced mTBI.
Post-concussion symptoms, memory, attention, visual and verbal functioning.
Symptom recovery, cognitive recovery.

\section{Neuropsychological} test performance (verbal learning, delayed memory, visual scanning, executive functioning, attention, concentration, information processing speed, bilateral fine motor speed, word fluency), post-concussion symptoms.

ImPACT, SCAT/ SCAT2; 11-12 (1-66); Daily (SCAT), once asymptomatic $(\operatorname{ImPACT})$.

HVLT, TMT A-B,Digit Span Test, SDMT, Grooved Pegboard Test, COWAT, PCSS; 4; within 24 h, 3, 5, 7 days $P C$.

Cognitive functioning, post-concussion symptoms.

Postural stability, post-concussion symptoms, cognitive performance.
ImPACT, BESS; 5; 2, 7, 14 days (ImPACT), 1, 2, 3 days (BESS).
ImPACT; 2; Up to 3 days, 7-10 days.
PCSC, HVLT, SDMT, Digit Span Test, Penn State Cancellation Test, TMT, COWAT, Stroop Test, Vigil Continuous Performance Test, five-word list-learning task; 4; 2, 48 h, 1 week, 1 month.

Locomotion, dual-task, cognitive function, information processing, attention, executive functioning, post-concussion symptoms. Keatley Symptom Questionnaire, modified Stroop test, walking kinematic lab measures,
Outcome scores not included; Symptoms not included; 12.5 days (symptom recovery) 21.1 days (cognitive recovery).

HVLT: 24.6 (4.0) TMT-A: 21.0 (5.9) TMT-B: 55.4 (17.3) Digit Span: 15.8 (3.9) SDMT:56.8 (8.9) Grooved Pegboard dominant/nondominant: 67.1 (10.7)/ 73.5 (11.9) COWAT: 37.5 (9.3), PCSS: 10.3 (12.6); Symptoms not included; Recovery not included.

Visual memory: 0.76, 0.74 Reaction time: 0.57, 0.53 Verbal memory: 0.85, 0.85 Processing speed: 38.81, 41.04 Total symptoms: $12.71,12.53$; Symptoms not included; Recovery not included.

BESS: 19.43 (1 day), 14.48 (3 days) Post concussion symptoms: 26.28 (2 days), 4.92 (14 days) Verbal memory processing: 80.89 (2 days), 82.26 (14 days) Visual memory: 67.18 (2 days), 68.15 (14 days) Reaction time: 0.648 (2 days), 0.578 (14 days) Motor processing speed: 36.52 (2 days), 40.74 (14 days); Symptoms not included; 7-14 days.

Symptom checklist: 8.53 (8.91) Digit span forward: $11.7(1.46)$ Hopkins learning index: $0.71(0.009)$ Hopkins delay index: $0.72(0.14)$ Hopkins percent retained: 87.85 (13.32) List learning immediate recall index: 0.98 (0.02); Headache, nausea, dizziness, balance problems, drowsiness, sensitivity to light and noise, memory problems, problems concentrating; Recovery not included.

Locomotion navigation task, Spatial and Digit Span Tests of Wechsler Memory Scale3rd edition, Brown-Peterson test, SDMT, TMT,
Maximum gait speed: $1.55 \mathrm{~m} / \mathrm{s}$ no obstacle, $1.51-1.53 \mathrm{~m} / \mathrm{s}$ with obstacle Stroop test with obstacle: 2-4 errors Minimum clearance: 0.3-0.58 Cognitive dual-task cost: no obstacle- 68.58 (42.98) with obstacle- 68.36-151.19 (55.87-121.18); Symptoms not included; Recovery not included. 
TABLE 2 | Continued

\begin{tabular}{|c|c|c|c|}
\hline $\begin{array}{l}\text { First Author, } \\
\text { Year; } \\
\text { Country; } \\
\text { Reference }\end{array}$ & $\begin{array}{l}\text { Study sample } \\
\text { characteristics (age } \\
\text { range/ mean in } \\
\text { years); gender; sport }\end{array}$ & $\begin{array}{l}\text { Pre-injury Evaluation } \\
\text { (Y/N); Control group } \\
\text { included? (Y/N); } \\
\text { Number of } \mathrm{mTBI} \\
\text { participants; } \\
\text { Definition of } \mathrm{mTBI}\end{array}$ & Outcomes studied \\
\hline
\end{tabular}

Field et al., 2003; USA (24)

Guty et al., 2020; USA

McCrea et al., 2013; USA (26)

McCrea et al., 2003; USA (27)

Meier et al., 2017; USA

(28)

Nelson et al., 2016; USA

(29)
High school and collegiate athletes; (20); 96\% male; football, women's soccer.
Y; Y; 35; Defined by American Academy of Neurology Practice Parameter. Diagnosed by sports medicine practitioner.

Y; N; 40; diagnosed by university sports medicine staff. soccer, football, lacrosse, basketball, wrestling, rugby.

Athletes; (17); 89\% male; football, soccer, lacrosse, ice hockey.

Collegiate athletes; (20); 100\% male; football.

Y; Y; 570; American Academy of Neurology grading. Diagnosed by athletic trainer or sport physician.

Y; Y; 94; An injury resulting from a blow to the head causing an alteration in mental status, and at least one symptom from the American Academy of Neurology Guideline for Management of Sports Concussion.

Diagnosed by sports physician or athletic trainer.

N; Y; 43; Diagnosed by sports medicine physician.

Y; Y; 618; Defined by American Academy of Neurology Practice Parameter. Diagnosed by sports medicine practitioner.
Symptoms, verbal learning \& memory, attention \&

concentration, speed of information processing, visual scanning \& executive functioning, word fluency, visual memory (hs only).

Cognitive functioning, memory, executive functioning, attention, processing speed, post-concussion symptoms.

Post-concussion symptoms, postural Stability, cognitive functioning.

Post-concussion symptoms, cognitive impairment, postural stability, neurocognitive functioning.

Behavior and cognitive function, mood, symptoms.

Symptoms, cognitive functioning, postural stability.
Tests used; Number of follow-ups: mean (range); Time of follow-up
Mean outcome scores; Common symptom presentation; Average recovery time
Color-word Interference Test of Delis-Kaplan Executive Function System, Test of Everyday Attention, California Computerized Assessment Package, Paced Auditory Serial Addition Test (neuropsychological tests); 2; 35-40 days (lab measures), neuropsychological test-w/in 14 days of lab measures.

PCSS, HVLT, Digit Span Test, SDMT, TMT A\&B, COWAT; 4; within $24 \mathrm{~h}, 3,5$, 7 days.

AWL: Total Immediate and Delay Recall, BVMT-R, SDMT, Digit Span Test, PSU Cancellation Test, Stroop Color-Word Test, BDI-FS, PCSS; 1; 6-36 months. GSC, BESS, SAC, HVLT, TMT Part B, SDMT, COWAT, Stroop test; 8; immediately, $3 \mathrm{~h}, 1,2,3,5,7, \& 45$ or 90 days.

GSC, SAC, BESS, HVLT, TMT-B, SDMT, COWAT, Stroop color-word test; 8; immediately, 3h, 1, 2, 3, 5, 7,90 days $\mathrm{PC}$.
HAM-D, HAM-A, ANAM; 3; 1 day, 1 week, 1 month.

GSC, SAC, BESS, HVLT, TMT-B, SDMT, Stroop test; 7 ; $3 \mathrm{~h}, 1,2,3,5,7, \& 45$ or 90 days.
Memory composite: 98.66 (10.55) Attention/executive functioning composite: 99.09 (6.52); Headache, irritability, sadness, fatigue, sleep disturbance; Recovery not included.

Only reported as odds ratio; Symptoms not included; $<7$ days.

Only reported as odds ratio; Balance deficits; 7 days.
Symptoms: 28.3 (24.5) HVLT total: 22.5 (4.3) HVLT delay: 6.5 (2.7);

Symptoms not included; 7 days. athletes; (20); 79\% basketball, soccer collegiate athletes; (16-20); 88\% male; football, soccer, lacrosse, hockey. 
TABLE 2 | Continued

\begin{tabular}{llllll}
\hline First Author, & $\begin{array}{l}\text { Study sample } \\
\text { characteristics (age }\end{array}$ & $\begin{array}{l}\text { Pre-injury Evaluation } \\
\text { (Y/N); Control group } \\
\text { Country; }\end{array}$ & $\begin{array}{l}\text { Outcomes studied } \\
\text { range/ mean in } \\
\text { included? }(\mathrm{Y} / \mathrm{N}) ;\end{array}$ & $\begin{array}{l}\text { Tests used; Number of } \\
\text { follow-ups: mean (range); }\end{array}$ & $\begin{array}{l}\text { Mean outcome scores; Common } \\
\text { symptom presentation; Average }\end{array}$ \\
& years); gender; sport & $\begin{array}{l}\text { Number of } \mathrm{mTBI} \\
\text { participants; } \\
\text { Definition of } \mathrm{mTBI}\end{array}$ & & \\
recovery time
\end{tabular}

\begin{tabular}{|c|c|c|c|c|c|}
\hline $\begin{array}{l}\text { Roiger et al., } \\
2015 \text { USA } \\
\text { (30) }\end{array}$ & $\begin{array}{l}\text { NCAA division } 1 \\
\text { athletes; (20); 100\% } \\
\text { male; football, }\end{array}$ & $\begin{array}{l}\text { Y; Y; 7; Diagnosed by } \\
\text { sports medicine } \\
\text { physician. }\end{array}$ & Depression. & $\begin{array}{l}\text { CES-D; } 3 ; 1 \text { week, } 1 \text { month, } \\
3 \text { months. }\end{array}$ & $\begin{array}{l}\text { CES-D: } 1 \text { week } 11.0,1 \text { month } 8.3,3 \\
\text { months } 6.4 \text {; Depression; }<1 \text { month. }\end{array}$ \\
\hline
\end{tabular}

\begin{tabular}{|c|c|c|c|c|}
\hline $\begin{array}{l}\text { Turner et al., } \\
\text { 2017; USA } \\
\text { (31) }\end{array}$ & $\begin{array}{l}\text { NCAA division } 1 \\
\text { athletes; (19); } 77 \% \\
\text { male; not described. }\end{array}$ & $\begin{array}{l}\mathrm{N} ; \mathrm{Y} ; 15 \text {; Diagnosed by } \\
\text { sports medicine } \\
\text { physician. }\end{array}$ & Mood, state anxiety. & $\begin{array}{l}\text { POMS, STAI; 3; within } 72 \mathrm{~h} \\
\text { day } 1 \text { of exercise, date of } \\
\text { return-to-play. }\end{array}$ \\
\hline
\end{tabular}

sports medicine

physician.
CES-D: 1 week 11.0, 1 month 8.3, 3

Tension: 2.21 (2.46) Anger: 1.43 (2.5) Fatigue: 5.14 (4.02) Depression: 1.64 (3.05) Vigor: 5.71 (6.02) Confusion: 3.29 (2.37) Total mood disturbance: 108.0 (15.48) State anxiety: 39.73 (12.84); Balance deficits; 7 days.

Values reported as predictors of depression; Depression; Recovery not included.

\begin{tabular}{|c|c|c|}
\hline $\begin{array}{l}\text { Vargas et al., } \\
\text { 2015; USA } \\
\text { (32) }\end{array}$ & $\begin{array}{l}\text { Collegiate athletes; } \\
(18) ; 77 \% \text { male; } \\
\text { football, lacrosse, } \\
\text { basketball, soccer, ice } \\
\text { hockey, wrestling. }\end{array}$ & $\begin{array}{l}\text { Y; Y; 84; Diagnosed by } \\
\text { sports physician or } \\
\text { athletic trainer. }\end{array}$ \\
\hline $\begin{array}{l}\text { Walton et al., } \\
\text { 2021; USA } \\
\text { (33) }\end{array}$ & $\begin{array}{l}\text { Collegiate athletes; } \\
\text { (19); } 45 \% \text { male; sport } \\
\text { not described. }\end{array}$ & $\begin{array}{l}\mathrm{N} ; \mathrm{Y} ; 20 \text {; diagnosed by } \\
\text { certified athletic trainer } \\
\text { based on Concussion } \\
\text { in Sport Group } \\
\text { definition. }\end{array}$ \\
\hline $\begin{array}{l}\text { Wright et al., } \\
2017 \text {; } \\
\text { Canada (34) }\end{array}$ & $\begin{array}{l}\text { Elite junior athletes; } \\
\text { (19); 100\% male; } \\
\text { hockey, football. }\end{array}$ & $\begin{array}{l}\text { Y; Y; 18; Diagnosed by } \\
\text { sports physician, based } \\
\text { on 4th International } \\
\text { Conference on } \\
\text { Concussion in Sport } \\
\text { consensus statement. }\end{array}$ \\
\hline $\begin{array}{l}\text { Zuckerman } \\
\text { et al., 2012; } \\
\text { US (35) }\end{array}$ & $\begin{array}{l}\text { High school and } \\
\text { collegiate athletes; (19); } \\
\text { 39\% male; football, } \\
\text { soccer, basketball, } \\
\text { softball. }\end{array}$ & $\begin{array}{l}\text { Y; N; 100; On-field } \\
\text { presentation of } \\
\text { post-concussive } \\
\text { symptoms. Diagnosed } \\
\text { by athletic trainer or } \\
\text { sports physician. }\end{array}$ \\
\hline
\end{tabular}

Depression.

BDI-FS, PHIQ, WTAR, PCSS, ImPACT; 1 ; 48 h- 41 days ( $71 \%$ within 5 days).

PCS, HRQOL, anxiety, fatigue, resilience, sleep disturbance.

Cerebral blood flow, symptom presence, symptom severity, balance, blood pressure.

Return to baseline, symptoms, cognitive function.
HIS-r, TBI-QOL, Neuro-QOL; 3 or 4; within $72 \mathrm{~h}, 10$ days, 17 days, after symptom free (if symptoms reported at third follow-up).

SCAT3, SAC, BESS; 3; 3 days, 2 weeks, 1 month.

ImPACT, PCSS; up to 2; 1-30 days.
Anxiety: 14.5 Resilience: 36 Stigma: 9 Sleep disturbance: 17.5 Fatigue: 22 Appetite: 0; Anxiety, resilience, stigma, sleep disturbance; 6 days (4-10) (symptom recovery) 14 days (10-16) (full participation).

Number of symptoms: 11 (5.8) Symptom severity: 25.6 (20.6) SAC: 26.2 (2.3) BESS: 4.2 (3.1); Symptoms not included; 14 (7-35) days.

Only includes baseline values; Symptoms not included; 5 days.

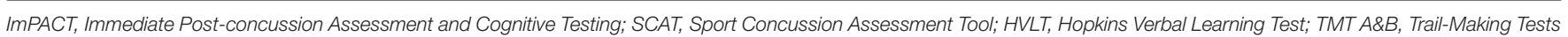

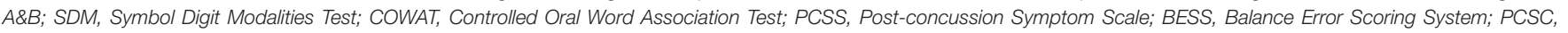

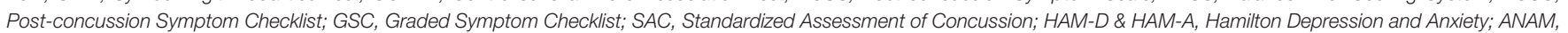

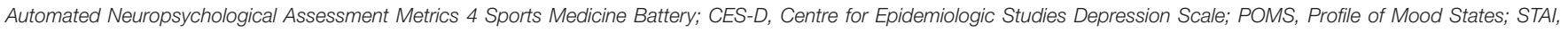
State-Trait Anxiety Inventory; BDI-FS, Beck Depression Inventory- Fast Screen; PHIQ, Previous Head Injury Questionnaire; WTAR, Wechsler Test of Adult Reading.

studies originated from the USA with the other three $(18,23,34)$ from Canada. The number of mTBI participants per study ranged from 6 to 570, with a median of 58 participants. The proportion of male participants in sport studies varied from 37 to $100 \%$, with more than $50 \%$ male participant groups in 13 of 18 studies. Four studies $(23,28,31,33)$ did not include pre-injury evaluations, and different four $(20,21,25,35)$ did not include a control or comparison group.

From the 24 general population mTBI articles, the mechanism of injury was most commonly from a fall or a motor vehicle accident. Five studies $(38,39,42,46,50)$ did not state the mechanism or setting of injury but did not relate incidents to sporting environments. Eleven studies (40, 42, 43, 45-47, 49, 53$55,57)$ included a control or a comparison group of differing injury severities. Almost half of included studies originated from the USA, UK, or Norway $(n=12)(12,38,39,42,44,48,50,52$, $53,55-57)$ with the remainder originating from various countries across North America, Europe, Oceania, and Asia. The number of participants included in each study that participated in each follow-up session ranged from 8 to 1,727 (median $=94$ ). Only three studies $(37,38,45)$ had $<50 \%$ male participant groups.

The most common outcomes measured for sports mTBI were post-concussion symptoms, depression, and cognitive functioning, while the most common outcomes assessed in non-sports mTBI studies were post-concussion symptoms, quality of life, cognitive and executive functioning, and personality or behavioral changes. To measure these outcomes, sports-related studies frequently used the Graded Symptom 
TABLE 3 | Non-sports-related studies.

\begin{tabular}{|c|c|c|c|c|c|}
\hline $\begin{array}{l}\text { First Author, } \\
\text { Year; } \\
\text { Country; } \\
\text { Reference }\end{array}$ & $\begin{array}{l}\text { Study sample } \\
\text { characteristics: (age } \\
\text { range/ mean in } \\
\text { years); gender; injury } \\
\text { setting }\end{array}$ & $\begin{array}{l}\text { Pre-injury Evaluation } \\
\text { (Y/N); Comparison } \\
\text { group included? } \\
\text { (Y/N); Number of } \\
\text { mTBI participants; } \\
\text { Definition of mTBI }\end{array}$ & Outcomes studied & $\begin{array}{l}\text { Tests used; Number } \\
\text { of follow-ups: mean } \\
\text { (range); Time of } \\
\text { follow-up }\end{array}$ & $\begin{array}{l}\text { Mean outcome scores; Common } \\
\text { symptom presentation; Average } \\
\text { recovery time }\end{array}$ \\
\hline $\begin{array}{l}\text { Beauchamp } \\
\text { et al., 2020; } \\
\text { Canada (36) }\end{array}$ & $\begin{array}{l}\text { Adults presenting at } \\
\text { emergency } \\
\text { departments; (23-57); } \\
61 \% \text { male; sking, } \\
\text { snowboarding, hockey, } \\
\text { soccer, football, motor } \\
\text { vehicle collision, bicycle } \\
\text { accident, pedestrian } \\
\text { accident. }\end{array}$ & $\begin{array}{l}\text { N; N; } 1727 ; \text { GCS } \\
13-15 .\end{array}$ & $\begin{array}{l}\text { Post-concussion } \\
\text { symptoms. }\end{array}$ & $\begin{array}{l}\text { RPCSQ; 3; 7, 30, } 90 \\
\text { days. }\end{array}$ & $\begin{array}{l}\text { Only reported as relative risks; } \\
\text { Headache, confusion, poor } \\
\text { concentration; Suggested within } 90 \\
\text { days. }\end{array}$ \\
\hline $\begin{array}{l}\text { Chiang et al., } \\
\text { 2016; Taiwan } \\
\text { (37) }\end{array}$ & $\begin{array}{l}\text { mTBI patients from a } \\
\text { neurosurgical } \\
\text { outpatient department; } \\
\text { (20-81); 49\% male; } \\
\text { MVA, Pedestrian hit by } \\
\text { vehicle, Fall, Hit by } \\
\text { object. }\end{array}$ & N; N; 100; GCS 13-15. & $\begin{array}{l}\text { Post-concussion } \\
\text { symptoms, quality of } \\
\text { life, outcome. }\end{array}$ & $\begin{array}{l}\text { CPCS, GOSE, } \\
\text { QOLIBRI, SF-36; 3; } 1 \text {, } \\
\text { 3, } 12 \text { months. }\end{array}$ & $\begin{array}{l}\text { Not included; Dizziness, fatigue, } \\
\text { headache, poor physical strength, } \\
\text { poor memory, poor concentration; } \\
54 \% \text { by } 1 \text { year }\end{array}$ \\
\hline $\begin{array}{l}\text { Cicerone } \\
\text { et al., 1995; } \\
\text { USA (38) }\end{array}$ & $\begin{array}{l}\text { Patients referred to a } \\
\text { neuropsychology clinic } \\
\text { due to persistent PCS; } \\
\text { (18-61); 38\% male; } \\
\text { unknown injury setting. }\end{array}$ & $\begin{array}{l}\mathrm{N} ; \mathrm{N} ; 50 \text {; Alteration of } \\
\text { mental status due to } \\
\text { injury characterized by } \\
\text { confusion, } \\
\text { posttraumatic amnesia } \\
\text { of }<24 \mathrm{~h} \text {, and loss of } \\
\text { consciousness of } \\
<30 \text { min. }\end{array}$ & $\begin{array}{l}\text { Post-concussion } \\
\text { symptoms, } \\
\text { neuropsychological } \\
\text { functioning, personality } \\
\text { and emotional } \\
\text { functioning, disability } \\
\text { status after injury. }\end{array}$ & $\begin{array}{l}\text { Post MTBI Symptom } \\
\text { Checklist, MMPI, } \\
\text { neuropsychological } \\
\text { testing battery } \\
\text { (attention, memory, } \\
\text { language, reasoning, } \\
\text { planning, organization); } \\
\text { 1; } 3-52 \text { months (m-14 } \\
\text { months). }\end{array}$ & $\begin{array}{l}\text { Not included; Irritability, frustration, } \\
\text { concentration, memory problems; } \\
51 \% \text { by } 1 \text { year. }\end{array}$ \\
\hline $\begin{array}{l}\text { Deb et al., } \\
\text { 1998; UK (39) }\end{array}$ & $\begin{array}{l}\text { Patients admitted to } \\
\text { hospital after minor } \\
\text { head injury; (18-93); } \\
67 \% \text { male; unknown } \\
\text { injury setting. }\end{array}$ & N; N; 137; GCS 13-15. & $\begin{array}{l}\text { Overall outcome, } \\
\text { physical disability, } \\
\text { cognitive state, } \\
\text { premorbid IQ, } \\
\text { psychiatric status, } \\
\text { post-concussion } \\
\text { symptoms. }\end{array}$ & $\begin{array}{l}\text { GOS, ERSS, MMSE, } \\
\text { NART, CIS-R, PSQ, } \\
\text { behavior rating scale; } \\
\text { 1; } 1 \text { year. }\end{array}$ & $\begin{array}{l}\text { Not included; Symptoms not } \\
\text { included; }<1 \text { year. }\end{array}$ \\
\hline $\begin{array}{l}\text { Emanuelson } \\
\text { et al., 2003; } \\
\text { Sweden (40) }\end{array}$ & $\begin{array}{l}\text { Patients attending the } \\
\text { Accident and } \\
\text { Emergency Unit if the } \\
\text { Department of General } \\
\text { Surgery; (16-60); } 65 \% \\
\text { male; Fall, hit by object, } \\
\text { alcohol abuse, traffic. }\end{array}$ & $\begin{array}{l}\mathrm{N} ; \mathrm{Y} ; 101 ;<30 \mathrm{~min} \text { loss } \\
\text { of consciousness. }\end{array}$ & $\begin{array}{l}\text { Quality of life, } \\
\text { symptoms, health, } \\
\text { well-being. }\end{array}$ & $\begin{array}{l}\text { SF-36, PCSC; 3; } 3 \\
\text { weeks, } 3 \text { months, } 1 \\
\text { year. }\end{array}$ & $\begin{array}{l}\text { At } 1 \text { year SF-36 scores- Physical } \\
\text { functioning: } 87.5 \text { Role physical: } 74.7 \\
\text { Bodily pain: } 72.2 \text { General health: } 70.9 \\
\text { Vitality: } 62.3 \text { Social functioning: } 83.2 \\
\text { Role emotional: } 77.2 \text { Mental health: } \\
74.9 \text { Physical composite score: } 49.1 \\
\text { Mental composite score: } 46.5 \\
\text { Number of symptoms at } 1 \text { year: } 0-18 \\
\text { (mean 3.75); Tiredness, headache, } \\
\text { neck pain, irritability, increased sleep, } \\
\text { depression, anxiety; Recovery time } \\
\text { not included }\end{array}$ \\
\hline $\begin{array}{l}\text { Fourtassi } \\
\text { et al., 2011; } \\
\text { Morocco (41) }\end{array}$ & $\begin{array}{l}\text { Head trauma patients } \\
\text { admitted to a teaching } \\
\text { hospital; (18-64); } 88 \% \\
\text { male; MVA, assault, fall, } \\
\text { sport. }\end{array}$ & N; N; 42; GCS 13-15. & $\begin{array}{l}\text { Post-concussion } \\
\text { symptoms, quality of } \\
\text { life. }\end{array}$ & $\begin{array}{l}\text { PCL, VAS; } 1 ;>1 \text { year } \\
\text { (m-15 months). }\end{array}$ & $\begin{array}{l}\text { Severity of symptoms from PCL: } \\
\text { difficulty remembering: } 2.38 \text { irritability: } \\
2.28 \text { fatigue: } 2.28 \text { noise sensitivity: } \\
2.28 \text { apathy: } 1.97 \text { headache: } 1.88 \\
\text { argumentative: } 1.69 \text { depression: } 1.54 \\
\text { anxiety: } 1.43 \text { doing things slowly: } \\
\text { 1.40; Difficulty remembering, } \\
\text { irritability, fatigue, noise sensitivity, } \\
\text { depression, argumentative, apathy, } \\
\text { headache, boredom, loneliness; }>1 \\
\text { year }\end{array}$ \\
\hline
\end{tabular}


TABLE 3 | Continued

\begin{tabular}{llllll}
\hline First Author, & Study sample & Pre-injury Evaluation & Outcomes studied & Tests used; Number & Mean outcome scores; Common \\
Year; & characteristics: (age & (Y/N); Comparison & & $\begin{array}{l}\text { of follow-ups: mean } \\
\text { (range); Time of }\end{array}$ & symptom presentation; Average \\
Country; & range/ mean in & group included? & & follow-up \\
Reference & years); gender; injury & (Y/N); Number of & & \\
& setting & mTBI participants; & & \\
& & Definition of $\mathrm{mTBI}$ & & \\
\end{tabular}

Hanks et al., Patients admitted to a 1999; USA Level 1 trauma centre;

(42)

(21); 73\% male

unknown injury setting.
N; Y; 138; GCS 13-15.

Emotional and behavioral changes, psychosocial adjustment.

N; Y; 37; GCS 13-15. Symptoms.

Heitger et al. 2007; New Zealand (43)

Hellstrøm et al., 2017; Norway (44)

Krpan et al. 2007;

Canada (46)
mTBI patients (15-56): 65\% male; Sports, MVA, bicycle accident, fall.
LecuyerGiguere

et al., 2019;

Canada (47)

Lucas et al., 2016; USA

(48)
TBI patients admitted to medical trauma centre; (34); 67\% male; unknown injury setting.

mTBI patients admitted to university-affiliated trauma-referral centre; (16-65); 68\% male; Traffic accident, fall, violence.

Adults from outpatient clinics; (40-55); 39\% male; traffic accident, ground-level fall, assault.

\section{mTBI patients} presenting to Emergency Room; (18-55); 60\% male; Sports, fall.

mTBI patients enrolled at a Level 1 trauma centre within 1 week of injury; (44); 76\% male; Vehicle, fall, violence, sports.
N; N; 62; GCS 13-15.

Post-concussion symptoms, global functioning, malingering, executive functioning.

N; Y; 110; diagnosed by neurosurgeon, GCS 14-15.

N; Y; 8; GCS 13-15. Coping strategies.

WOC-R; $1 ; 1$ year.

Executive functioning memory, information processing, depression, anxiety, irritability.

RPQ, GOSE, FIT, WAIS-III, CWIT, FAS; 2 4 weeks, 12 months.

TWSLT, VFT, PASAT-R, BDI-II, BAI, NTUIS, CPCS; 2; 2 weeks, 3 years.

HFUQ, SF-36v2; 4; 1 week, 3 , 6, 12 months.

niffin' Sticks Inventory Test, UPSIT, RBANS, DKEFS, TMT A-B, WAIS-IV, HADS, RPQ; 2; $24 h, 1$ year.

N; N; 212; GCS 13-15.

Olfactory function cognitive function, executive function, affective status, post-concussion symptoms.

Prevalence of headache, depression, comorbid headache and depression.
PHQ-9; 2; 1 week, 1 year.

KAS- anxiety: 8.28 belligerence: 5.92 sensory-perceptual distortions: 6.54 confusion: 3.97 helplessness: 6.48 hyperactivity: 4.69 negativity: 14.51 nervousness: 7.47 general psychopathology: 39.44 stability: 27.04 suspiciousness: 6.37 poor self-monitoring: 7.18 withdrawal: 10.53; Anxiety, belligerence, sensory-perceptual difficulties, confusion, helplessness, hyperactivity, negativism, nervousness, general adjustment difficulties; $>1$ year.

$\mathrm{RPQ}$ at 1 year- headache: 0.8 dizziness: 0.7 nausea: 0.2 noise sensitivity: 0.5 sleep disturbance: 0.7 fatigue: 0.9 irritability: 0.6 depression: 0.5 frustration: 0.6 poor memory: 0.8 poor concentration: 1.0 slowed thinking: 0.7 blurred vision: 0.7 light sensitivity: 0.5 double vision: 0.3 restlessness: 0.5 ; poor concentration, fatigue, taking longer to think, poor memory, headache; 3 months.

RPQ total: 13.8 (15.2), 14.0 (13.1) GOSE: 7.0 (.856), 7.14 (.848); Symptoms not included; Recovery time not included.

Long-term (mean: 2.9 years range: 6 months-6 years) follow-up CPCSPhysical: 4.21 Cognitive: 1.86 Emotional: 1.26 Total: 7.38; fatigue, loss of energy, insomnia, slowness of information processing, irritability, blurred vision; By long-term follow-up date.

WOC-R confrontive: 4.5 distancing: 6.0 self-controlling: 10.0 seeking social support: 5.1 accepting responsibility: 4.1 escape-avoidant: 6.6 planful problem-solving: 8.1 positive reappraisals: 5.8 total score: 8.1; Symptoms not included; Recovery time not included.

UPSIT: 34.0, sniffin' sticks TDI at baseline: 31.1; hyposmia; >1year.

Not included; headache, depression; Suggested increase in symptoms at 1 year. 
TABLE 3 | Continued

\begin{tabular}{llllll}
\hline First Author, & Study sample & Pre-injury Evaluation & Outcomes studied & Tests used; Number & Mean outcome scores; Common \\
Year; & characteristics: (age & (Y/N); Comparison & & $\begin{array}{l}\text { of follow-ups: mean } \\
\text { (range); Time of }\end{array}$ & symptom presentation; Average \\
Country; & range/ mean in & group included? & & follow-up \\
Reference & years); gender; injury & (Y/N); Number of & & \\
& setting & mTBI participants; & & \\
& & Definition of mTBI & & \\
\end{tabular}

Losoi et al., $\quad$ mTBI patients N; Y; 74; GCS 14-15.

2016; Finland presenting at

(49) emergency department of hospital; (37); 61\% male; Fall, sports, MVA, bicycle accident.

\begin{tabular}{|c|c|}
\hline $\begin{array}{l}\text { McMahon } \\
\text { et al., 2014; } \\
\text { USA (50) }\end{array}$ & $\begin{array}{l}\text { Patients at one of three } \\
\text { Level } 1 \text { trauma } \\
\text { centres;(18-94); } 70 \% \\
\text { male; unknown injury } \\
\text { setting. }\end{array}$ \\
\hline $\begin{array}{l}\text { Nelson et al., } \\
\text { 2019; USA } \\
\text { (12) }\end{array}$ & $\begin{array}{l}\text { Patients presenting to } \\
\text { level } 1 \text { trauma centres; } \\
\text { (Mean age 41); (66\% } \\
\text { male); Vehicle } \\
\text { accidents, fall, assault } \\
\text { and other. }\end{array}$ \\
\hline $\begin{array}{l}\text { Oldenburg } \\
\text { et al., 2018; } \\
\text { Sweden (51) }\end{array}$ & $\begin{array}{l}\text { Patients at any of three } \\
\text { emergency } \\
\text { departments; (15-65); } \\
61 \% \text { male; Fall, traffic, } \\
\text { assault. }\end{array}$ \\
\hline
\end{tabular}

N; N; 375; GCS 13-15. Post-concussion symptoms, post-TBI outcome.

Post-concussion symptoms, fatigue, insomnia, pain, post-traumatic stress, depression, quality of life, resilience, return-to-work.

N; Y; 1154; GCS 13-15.

N; N; 94; GCS 13-15.

Post-concussion symptoms, neurological disorders, stress reaction, preinjury behavior and personality.

Functioning, post-concussion symptoms, psychological distress. symptoms.

2009; Norway (52)

Patients enrolled at the neurosurgical department of a hospital; (16-60); 62\% male; Traffic, fall, violence.

Sigurdardottir et al., 2009; Norway (53)
Patients at a Level 1 trauma centre; (16-55); $62 \%$ male; Traffic, fall, assault.
N; N; 96; GCS 13-15. Post-concussion

N; Y; 40; GCS 13-15.

Post-concussion symptoms, neuropsychological functioning.

Tasmanian

et al., 2013:

Australia (54)

Neurotrauma Register;

(16-83); 61\% male;

Transport-related; fall, assault, sport.

Steward Patients from University

et al., 2016; of Alabama School of

USA (55) Medicine; (19-79); 55\%

male; MVA, fall, other

vehicle accident.
N; N; 172; GCS 13-15. Full Scale IQ, anxiety, depression.

NART, HADS; 3; 1, 6, 12 months.

Medical decision-making capacity, capacity to consent, reasoning, understanding months
GOSE, RPQ, BSI; 4; 2 weeks, 3, 6, 12

months.

SI, Pain Subscle of the RNBI, PTSD

Checklist- Civilian version, BDI-II, RS, QOLIBRI, RAVLT, Stroop test, TMT A-B, Finger Tapping Test, WAIS-III, RTW; 3; 1, 6, 12 months.

GOSE, BSI-18, RPQ, SWLS,PCSC; $3 ; 3,6$, 12 months.

$R P Q, R H F \cup Q$, Axis I-V of DSM-IV, HADS, IES-R, SSP, SOC, AUDIT; $1 ; 1$ week, 1 year.

RPQ; 4; Within 48 h, 3, 6, 12 months.

At 1 year- RPCSQ: 6.9 BNI-FS: 8.1 ISI: 4.3 RNBI: 7.3 PCL-C: 23.3 RS: 143.3 QOLIBRI: 158.4 SWLS: 27; fatigue, insomnia, pain; $73.3 \%$ by 1 year.

At 1 year- total PCS symptoms: 6.8 physical symptoms: 2.8 cognitive symptoms: 1.7 emotional symptoms: 1.3 sleep symptoms: 1.1; Symptoms not included; Suggested increase in some symptoms at 1 year

Only reported as percentage prevalence; headache, fatigue, depression, forgetfulness; $47.2 \%$ by 1 year.

At 1 year for recovered group- IES-R: intrusions: 6.2 avoidance: 4.8 hyperarousal: 3.5 total: 14.5 HADS: anxiety: 2.7 depression: 2.2 for group reporting symptoms at 1 year- IES-R: intrusions: 15.2 avoidance: 9.3 hyperarousal: 12.4 total: 36.8 HADS: anxiety: 8.4 depression: 6.7; Symptoms not included; $88 \%$ symptom-free at 1 year.

Not included; Headache, dizziness, fatigue, noise sensitivity, sleep disturbance, forgetfulness, poor concentration; Suggested increase in some symptoms at 1 year.

RPQ, HADS, GOAT; 3; Within $24 \mathrm{~h}, 3,12$ months.

At 3 months- RPQ: 20.8 (18.3) HADS: 6.9 (4.9) At 1 year- RPQ: 15.9 (16.9) HADS: 5.4 (4.6); Headache, fatigue, frustration, memory problems, concentration problems, taking longer to think, restlessness; $72.7 \%$ by 1 year.

Estimated FSIQ at 1 year- 106.28 (8.47); Symptoms not included; $<6$ months.

CCTI, GOAT; 3; 1, 6, 12

GOAT at 1 year- mTBI: 93.08 complicated mTBI: 97.38. CCTI at 1 year- mTBI [complicated mTBI]: expressing choice: 4.0 [3.94] reasonable choice: 0.96 [1.0] 
TABLE 3 | Continued

\begin{tabular}{|c|c|c|c|c|c|}
\hline $\begin{array}{l}\text { First Author, } \\
\text { Year; } \\
\text { Country; } \\
\text { Reference }\end{array}$ & $\begin{array}{l}\text { Study sample } \\
\text { characteristics: (age } \\
\text { range/ mean in } \\
\text { years); gender; injury } \\
\text { setting }\end{array}$ & $\begin{array}{l}\text { Pre-injury Evaluation } \\
\text { (Y/N); Comparison } \\
\text { group included? } \\
\text { (Y/N); Number of } \\
\text { mTBI participants; } \\
\text { Definition of } \mathrm{mTBI}\end{array}$ & Outcomes studied & $\begin{array}{l}\text { Tests used; Number } \\
\text { of follow-ups: mean } \\
\text { (range); Time of } \\
\text { follow-up }\end{array}$ & $\begin{array}{l}\text { Mean outcome scores; Common } \\
\text { symptom presentation; Average } \\
\text { recovery time }\end{array}$ \\
\hline
\end{tabular}

\begin{tabular}{|c|c|c|c|c|}
\hline $\begin{array}{l}\text { Theadom } \\
\text { et al., 2016; } \\
\text { New Zealand } \\
\text { (13) }\end{array}$ & $\begin{array}{l}\text { mTBI patients residing } \\
\text { in Hamilton or Waikato } \\
\text { districts; (38); } 59 \% \\
\text { male; Fall, MVA, } \\
\text { exposure to } \\
\text { mechanical force, } \\
\text { assault. }\end{array}$ & N; N; 342; GCS 13-15. & $\begin{array}{l}\text { Post-concussion } \\
\text { symptoms, quality of } \\
\text { life, cognitive } \\
\text { functioning, } \\
\text { depression, anxiety, } \\
\text { overall functioning. }\end{array}$ & $\begin{array}{l}\text { RPQ, GOS, CNS-VS } \\
\text { HADS, SF-36; 4; witl } \\
2 \text { weeks, 1, 6, } 12 \\
\text { months. }\end{array}$ \\
\hline $\begin{array}{l}\text { Singh et al., } \\
\text { 2019; UK (56) }\end{array}$ & $\begin{array}{l}\text { TBI patients presenting } \\
\text { to emergency } \\
\text { department of teaching } \\
\text { hospital; (17-94); } 69 \% \\
\text { male; Fall, traffic, } \\
\text { assault. }\end{array}$ & $\mathrm{N} ; \mathrm{N} ; 651 ;$ GCS 12-15 & $\begin{array}{l}\text { Post-concussion } \\
\text { symptoms, } \\
\text { return-to-work, } \\
\text { functioning, global } \\
\text { outcome. }\end{array}$ & $\begin{array}{l}\text { GOSE, RHFUQ, HAL } \\
\text { RPQ, RTW; 2; } 10 \\
\text { weeks, } 1 \text { year. }\end{array}$ \\
\hline $\begin{array}{l}\text { Sterr et al., } \\
\text { 2006; UK (57) }\end{array}$ & $\begin{array}{l}\text { mTBI patients } \\
\text { attending one of } 150 \\
\text { general practitioner } \\
\text { surgeries, a local Brain } \\
\text { Injury Community } \\
\text { Centre, or a University } \\
\text { campus; (18-65); 63\% } \\
\text { male; Fall, sport, traffic, } \\
\text { assault. }\end{array}$ & $\begin{array}{l}\mathrm{N} ; \mathrm{Y} ; 38 \text {; Loss of } \\
\text { consciousness } \\
<30 \text { min, post } \\
\text { traumatic amnesia } \\
<24 \mathrm{~h} \text {, alteration of } \\
\text { mental state (dazed, } \\
\text { disorientated, } \\
\text { confused) at time of } \\
\text { incident. }\end{array}$ & $\begin{array}{l}\text { Post-concussion } \\
\text { symptoms, cognitive } \\
\text { functioning, attention, } \\
\text { IQ. }\end{array}$ & $\begin{array}{l}\text { RPQ, CFQ, TAP, } \\
\text { CANTAB, NART; 1; } \\
\text { 12+ months. }\end{array}$ \\
\hline
\end{tabular}

appreciation: 7.08 [7.13] reasoning: 8.81 [8.13] understanding: 61.23 [60.94]; Poor appreciation, reasoning, and understanding; 6-12 months Reported as percentage/number of participants; headache, fatigue, forgetfulness, poor concentration, taking longer to think; >50\% by 1 year.

GOSE at 1 year (number of participants): dead: 20 severe lower: 1 severe upper: 38 moderate lower: 88 moderate upper: 109 good lower: 129 good upper: 215. Return-to-work at 1 year- full: 345 partial: 149 none: 86; Symptoms not included; Recovery not included. Only included in graphic form; Headache, noise sensitivity, sleep disturbance, fatigue, irritability, depression, frustration, memory issues, poor concentration, taking longer to think; $71 \%$ within 1 year.

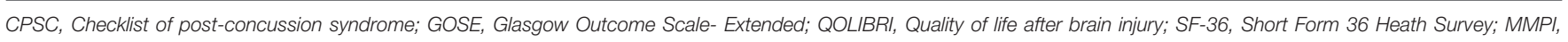

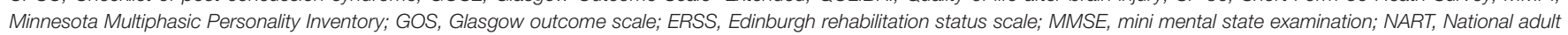

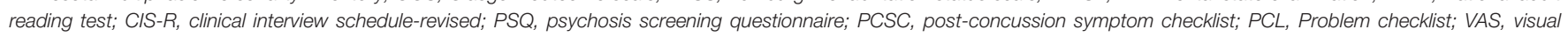

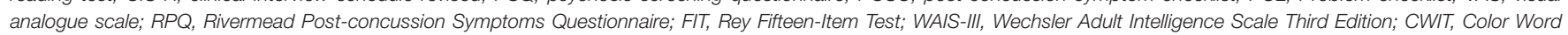

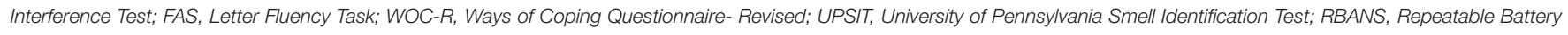

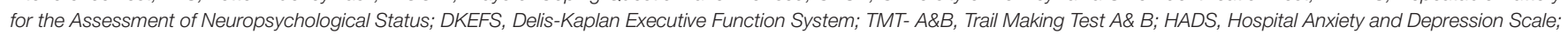

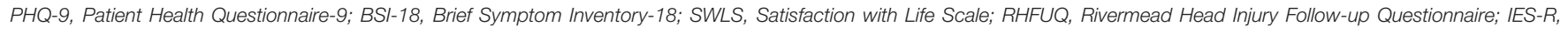

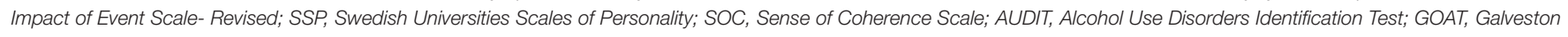

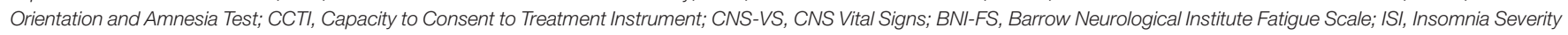

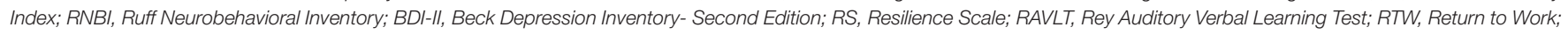
KAS, Katz Adjustment Scale; CFQ, Cognitive Failures Questionnaire; TAP, Test of Attentional Performance; CANTAB, Cambridge Neuropsychological Test Automated Battery.

Checklist (GSC), Post-concussion Symptom Scale (PCSS), and the Immediate Post-Concussion Assessment and Testing (ImPACT), while general population studies used the Rivermead Post-concussion Symptoms Questionnaire (RPQ), Glasgow Outcome Scale [Extended] (GOS/GOSE), Hospital Anxiety and Depression Scale (HADS), and the 36-Item Short Form Survey (SF-36).

The mean number of follow-up sessions in general population studies was two, while the sport studies averaged four followup time points. Only six general population studies $(43,45,47$, $48,51-53$ ) conducted the first follow-up appointment within 1 week of injury, while fifteen of the sports mTBI studies conducted their first follow-up within this time frame. Time of follow-ups ranged from $<3 \mathrm{~h}$ to 36 months post-mTBI in the sports studies, with 11 studies $(22,23,25-30,32,34,35)$ including a follow-up testing session at least 1 -month post-injury or later. Of those 11 , only six studies $(23,25-27,29,30)$ included the latest follow-up time point of 90 days or 3 months. All but 1 of the 24 general population studies (36) included a follow-up at 12 months or later, with the earliest follow-up within $24 \mathrm{~h}$ post-injury. The most frequent follow-up times $<12$ months were at 1 month $(n$ $=8)$ and 6 months $(n=8)$.

In the sports studies, the mean age of participants ranged from 17 to 20 , while the general population studies mean age was older at 30-40 years. Of the five non-sport studies $(36,43,47,49,57)$ that included sports as a mechanism of injury in at least $10 \%$ of participants, only one study (36) directly compared outcomes of sports vs. non-sports-related mTBI participants. No comparison 
was made by other authors between groups of sports vs. nonsport, and the participants were not differentiated or split in data in a way to enable these comparisons to be made. The sole study comparing sports vs. non-sports (36) investigated prevalence of post-concussion symptoms and return to daily activities at 7 - and 90-day post-mTBI based on results from the Rivermead Postconcussion Questionnaire. This study revealed no differences in overall symptom burden, mood, or recovery at 7 - and 90-days post-injury (36).

Due to the lack of comparable data due to use of different outcome measures and follow-up time frames, we were not able to compare disease burden or recovery time frames between the two contexts apart from using data obtained within one study (36).

\section{DISCUSSION}

This scoping review highlighted some key methodological differences in how studies are conducted between sports and non-sports related mTBI, making comparisons in symptom presentation and impact between the two contexts difficult. The review has highlighted a significant gap in the current literature, given the key differences in current evidence for the definitions used, outcome measures used to assess impact such as symptoms and cognitive function, and timing of outcome assessments. There is a need for collaboration and consensus between the two fields to enable greater understanding of the similarities and differences of sports- and non-sports-related injuries to guide clinical decision-making.

One key difference between the two contexts was the way injuries were described and classified. In the non-sports context, mTBIs were assessed and framed in terms of injury severity by GCS score, whereas, in contrast, there was no reference to the broader context or how it was decided this was a "concussive" injury and not of moderate severity within the sports context. Consequently, it was not clear which types of injuries were excluded from mild SR-TBI studies. Given moderate to severe injuries can be sustained within sports context (59), this may be something that sports physicians and academics consider, record, and report on. In updated versions of commonly used SR-TBI assessments, such as the SCAT-5, one portion of the assessment tool includes conducting the GCS (60). Given ambulance personnel routinely use GCS in most countries, this could easily be reported on SR-TBI studies to assist in study comparison.

One consideration in conducting this review was that, often, general population studies included SR-TBI within a larger dataset. However, these injuries were often classified under different mechanism categories (e.g., falls and being hit by an object), whereas SR-TBI studies focused on injuries specifically on any injury sustained during sport. It was not possible to combine data from these studies to explore differences in symptom presentation and recovery time frames due to the methodological and reporting differences. For example, general population studies presented data that between 47 and $88 \%$ of participants had recovered by 1 year. In contrast, the sportsrelated studies reported findings that average days to recovery were between 7 and 14 days. However considerable caution needs to be taken to directly interpret these findings as the way recovery was defined and measured was not comparable and restricted by few longer-term follow-ups in sports-related mTBI studies. The only direct comparison (36) made between sports- and nonsports-related mTBI groups found few differences in symptom presentation between groups. Whilst there were no differences in overall symptom burden or recovery between the two groups in this study, on a symptom-specific level, SR-TBI participants were at a greater risk of impaired concentration compared to nonsports participants in the week following mTBI, and exhibited fewer and less severe symptoms such as fatigue and dizziness at the later follow-up. This suggested that there may be small discrepancies between sports and non-sports mTBI presentation for some symptoms. However, additional studies are needed to compare symptom presentation and recovery time frames between the two contexts to determine if these injuries do need to be considered separately or whether a blended model, with embedded flexibility, may be a more effective and simpler health care pathway.

Additionally, discrepancy between studies within the two contexts was the lack of long-term follow-ups in sports-related mTBI studies compared to studies on the general population. Previous epidemiology research (13) showed significant prevalence of post-concussion symptoms up to 12 months post-mTBI in a community population including sports-related injuries. However, this review found that individuals who had suffered an SR-TBI were very rarely assessed for any mTBIrelated outcomes beyond 3 months post-injury. Whilst many patients do recover relatively quickly following an mTBI (5), there is likely to be a proportion who experiences an SR-TBI who may still experience difficulties beyond 3 months, particularly if there was significant mTBI history. There is evidence that those who have experienced prior injuries have been more at risk of a prolonged recovery (61). Case studies were presented where sports athletes were report long-term and cumulative impacts (62). Increasing follow-up length in SR-TBI-related studies would help identify risk factors in longer-term problems and key intervention points in recovery trajectories where more intensive support is required to facilitate recovery. Although not conclusive, the findings from this review highlighted that information about possible long-term effects of an mTBI was lacking within the sports context.

There was a considerable overlap in the types of outcome domains studies, e.g., symptom presentation, cognitive function, and mood; however, the diversity in outcome measures used to assess these domains prevented direct comparison of the findings. Whilst there is a clear benefit in researching specific subpopulations in detail; there is also a need to look more broadly across the disorder spectrum. This review highlights a need for integration and collaboration across these two fields of enquiry to advance the field. The neurophysiological trauma to the brain is likely similar no matter whether the mechanisms are from sports-related or non-sports-related activities. Therefore, further evidence is needed to determine if different approaches to 
assessment and management should be similar or different across injury contexts.

\section{Limitations}

The key limitation of this review related to the search terms included. Overly broad terms such as "outcome" or "recovery" were not included to decrease the high number of irrelevant articles that were identified when including these terms. However, this may have inadvertently resulted in the exclusion of relevant studies. Since completion of the review, other relevant studies have been published but were not able to be considered within this review such as the Concussion Assessment, Research, and Education Consortium research (63). However, the key differences in methods used between these two fields of study are important to highlight to guide future research and to ensure optimal health care pathways are developed to support patients most effectively. Additionally, the sample size for some studies was low. Once the gap in the current literature has been addressed, it will be important to conduct a systematic review including of study quality to identify if there are similarities and differences in self-reported recovery following sports and non-sports-related injuries.

\section{CONCLUSION}

This review highlighted a key gap in the current literature, comparing recovery trajectories across different contexts of

\section{REFERENCES}

1. Menon DK, Schwab K, Wright DW, Maas AI. Position statement: definition of traumatic brain injury. Arch Phys Med Rehabil. (2010) 91:163740. doi: 10.1016/j.apmr.2010.05.017

2. Harmon KG, Drezner J, Gammons M, Guskiewicz K, Halstead M, Herring S, et al. American medical society for sports medicine position statement: concussion in sport. Clin J Sport Med. (2013) 23:1-18. doi: 10.1097/JSM.0b013e31827f5f93

3. West TA, Marion DW. Current recommendations for the diagnosis and treatment of concussion in sport: a comparison of three new guidelines. $J$ Neurotrauma. (2014) 31:159-68. doi: 10.1089/neu.2013.3031

4. Sharp DJ, Jenkins PO. Concussion is confusing us all. Pract Neurol. (2015) 15:172-86. doi: 10.1136/practneurol-2015-001087

5. McCrory P, Meeuwisse W, Dvorák J, Aubry M, Bailes J, Broglio S, et al. Consensus statement on concussion in sport-the 5th international conference on concussion in sport held in Berlin, October 2016. Br J Sports Med. (2017) 51:838-47. doi: 10.1136/bjsports-2017-097699

6. Hume PA, King D, McGeown J, Theadom A. Sports-related concussion, mild traumatic brain injury or sport-originated brain injury (SOBI): a more useful term. N Z J Sports Med. (2019) 45:64-7.

7. Maas AIR, Menon DK, Adelson PD, Andelic N, Bell MJ, Belli A, et al. Traumatic brain injury: integrated approaches to improve prevention, clinical care, and research. Lancet Neurol. (2017) 16:987-1048. doi: 10.1016/S1474-4422(17)30371-X

8. Dewan MC, Rattani A, Gupta S, Baticulon RE, Hung YC, Punchak M, et al. Estimating the global incidence of traumatic brain injury. J Neurosurg. (2018) 130:1-1097. doi: 10.3171/2017.10.JNS17352

9. Feigin VLP, Theadom AP, Barker-Collo SP, Starkey NJP, McPherson $\mathrm{KP}$, Kahan MMB, et al. Incidence of traumatic brain injury in New Zealand: a population-based study. Lancet Neurol. (2013) 12:53-64. doi: 10.1016/S1474-4422(12)70262-4 injury and has reflected a need for greater collaboration between sports and general population fields of research. Future research should focus on longer-term follow-ups, particularly in sports-related studies, and utilize a more standardized method of assessing mTBI- affected individuals to inform optimal treatment approaches.

\section{DATA AVAILABILITY STATEMENT}

The original contributions presented in the study are included in the article/supplementary material, further inquiries can be directed to the corresponding author.

\section{AUTHOR CONTRIBUTIONS}

All authors listed have made a substantial, direct, and intellectual contribution to the work and approved it for publication.

\section{FUNDING}

This review was conducted with support from an AUT, Faculty of Health and Environmental Sciences Summer Scholarship Grant. The funder was not involved in the study design or interpretation of the findings. Funds for open access publication fees were provided by the AUT TBI Network.

10. Cnossen M, Polinder S, Allanson J, Anke A, Audibert G, Badenes R, et al. Variation in structure and process of care in traumatic brain injury: provider profiles of European neurotrauma centers participating in the CENTER-TBI study. PLoS ONE. (2016) 11:e0161367. doi: 10.1371/journal.pone.0161367

11. Marshall S, Bayley M, McCullagh S, Berrigan L, Fischer L, Ouchterlony D, et al. Guideline for Concussion/Mild Traumatic Brain Injury and Persistent Symptom (for Adults $18+$ years of age). 3rd ed Toronto, ON: Ontario Neurotrauma Foundation (2018).

12. Nelson LD, Temkin NR, Dikmen S, Barber J, Giacino JT, Yuh E, et al. Recovery after mild traumatic brain injury in patients presenting to US level i trauma centers: a transforming research and clinical knowledge in traumatic brain injury (TRACK-TBI) study. JAMA Neurol. (2019) 76:104959. doi: 10.1001/jamaneurol.2019.1313

13. Theadom A, Parag V, Dowell T, McPherson K, Starkey N, Barker-Collo $S$, et al. Persistent problems 1 year after mild traumatic brain injury: a longitudinal population study in New Zealand. Br J Gen Pract. (2016) 66:e1623. doi: 10.3399/bjgp16X683161

14. Möller MC, Lexell J, Wilbe Ramsay K. Effectiveness of specialized rehabilitation after mild traumatic brain injury: a systematic review and metaanalysis. J Rehabil Med. (2021) 53:jrm00149. doi: 10.2340/16501977-2791

15. Klein AP, Tetzlaff JE, Bonis JM, Nelson LD, Mayer AR, Huber DL, et al. Prevalence of potentially clinically significant magnetic resonance imaging findings in athletes with and without sport-related concussion. $J$ Neurotrauma. (2019) 36:1776-85. doi: 10.1089/neu.2018.6055

16. Manley G, Gardner AJ, Schneider KJ, Guskiewicz KM, Bailes J, Cantu RC, et al. A systematic review of potential long-term effects of sport-related concussion. Br J Sports Med. (2017) 51:969-77. doi: 10.1136/bjsports-2017097791

17. Munn Z, Peters MDJ, Stern C, Tufanaru C, McArthur A, Aromataris E. Systematic review or scoping review? guidance for authors when choosing between a systematic or scoping review approach. BMC Med Res Methodol. (2018) 18:143. doi: 10.1186/s12874-018-0611-x 
18. Black AM, Sergio LE, Macpherson AK. The epidemiology of concussions: number and nature of concussions and time to recovery among female and male canadian varsity athletes 2008 to 2011. Clin J Sport Med. (2017) 27:52-6. doi: 10.1097/JSM.0000000000000308

19. Collins MW, Grindel SH, Lovell MR Dede DE, Moser DJ, Phalin $\mathrm{BR}$, et al. Relationship between concussion and neuropsychological performance in college football players. JAMA. (1999) 282:96470. doi: 10.1001/jama.282.10.964

20. Covassin T, Schatz P, Swanik CB. Sex differences in neuropsychological function and post-concussion symptoms of concussed collegiate athletes. Neurosurgery. (2007) 61:345-50. doi: 10.1227/01.NEU.0000279972.95060.CB

21. Covassin T, Elbin RJ, Harris W, Parker T, Kontos A. The role of age and sex in symptoms, neurocognitive performance, and postural stability in athletes after concussion. Am J Sports Med. (2012) 40:130312. doi: $10.1177 / 0363546512444554$

22. Echemendia RJ, Putukian M, Mackin RS, Julian L, Shoss N. Neuropsychological test performance prior to and following sportsrelated mild traumatic brain injury. Clin J Sport Med. (2001) 11:23-31. doi: 10.1097/00042752-200101000-00005

23. Fait P, Swaine B, Cantin JF, Leblond J, McFadyen BJ. Altered integrated locomotor and cognitive function in elite athletes 30 days postconcussion: a preliminary study. J Head Trauma Rehabil. (2013) 28:293-301. doi: 10.1097/HTR.0b013e3182407ace

24. Field M, Collins MW, Lovell MR, Maroon J. Does age play a role in recovery from sports-related concussion? a comparison of high school and collegiate athletes. J Pediatr. (2003) 142:546-53. doi: 10.1067/mpd.2003.190

25. Guty E, Riegler K, Meyer J, Walter AE, Slobounov SM, Arnett P. Symptom factors and neuropsychological performance in collegiate athletes with chronic concussion symptoms. Arch Clin Neuropsychol. (2020) 36: 74656. doi: 10.1093/arclin/acaa092

26. McCrea M, Guskiewicz K, Randolph C, Barr WB, Hammeke TA, Marshall SW, et al. Incidence, clinical course, and predictors of prolonged recovery time following sport-related concussion in high school and college athletes. J Int Neuropsychol Soc. (2013) 19:22-33. doi: 10.1017/S1355617712000872

27. McCrea M, Guskiewicz KM, Marshall SW, Barr W, Randolph C, Cantu $\mathrm{RC}$, et al. Acute effects and recovery time following concussion in collegiate football players: the NCAA concussion study. JAMA. (2003) 290:255663. doi: 10.1001/jama.290.19.2556

28. Meier TB, Bellgowan PSF, Mayer AR. Longitudinal assessment of local and global functional connectivity following sports-related concussion. Brain Imaging Behav. (2017) 11:129-40. doi: 10.1007/s11682-016-9520-y

29. Nelson LD, Guskiewicz KM, Barr WB, Hammeke TA, Randolph C, Ahn $\mathrm{KW}$, et al. Age differences in recovery after sport-related concussion: a comparison of high school and collegiate athletes. J Athl Train. (2016) 51:14252. doi: 10.4085/1062-6050-51.4.04

30. Roiger T, Weidauer L, Kern B. A longitudinal pilot study of depressive symptoms in concussed and injured/nonconcussed national collegiate athletic association division i student-athletes. J Athl Train. (2015) 50:25661. doi: 10.4085/1062-6050-49.3.83

31. Turner S, Langdon J, Shaver G, Graham V, Naugle K, Buckley T. Comparison of psychological response between concussion and musculoskeletal injury in collegiate athletes. Sport Exerc Perform Psychol. (2017) 6:277-88. doi: 10.1037/spy0000099

32. Vargas G, Rabinowitz A, Meyer J, Arnett PA. Predictors and prevalence of postconcussion depression symptoms in collegiate athletes. J Athl Train. (2015) 50:250-5. doi: 10.4085/1062-6050-50.3.02

33. Walton SR, Broshek DK, Kranz S, Malin SK, Hertel J, Resch JE. Mood, psychological, and behavioral factors of health-related quality of life throughout recovery from sport concussion. J Head Trauma Rehabil. (2021) 36:128-36. doi: 10.1097/HTR.0000000000000604

34. Wright AD, Smirl JD, Bryk K, van Donkelaar P. A prospective transcranial doppler ultrasound-based evaluation of the acute and cumulative effects of sport-related concussion on neurovascular coupling response dynamics. $J$ Neurotrauma. (2017) 34:3097-106. doi: 10.1089/neu.2017.5020

35. Zuckerman SL, Lee YM, Odom MJ, Solomon GS, Forbes JA, Sills AK. Recovery from sports-related concussion: days to return to neurocognitive baseline in adolescents versus young adults. Surg Neurol Int. (2012) 3:130. doi: 10.4103/2152-7806.102945
36. Beauchamp F, Boucher V, Neveu X, Ouellet V, Archambault P, Berthelot S, et al. Post-concussion symptoms in sports-related mild traumatic brain injury compared to non-sports-related mild traumatic brain injury. CJEM. (2021) 23:223-31. doi: 10.1007/s43678-020-00060-0

37. Chiang CC, Guo SE, Huang KC, Lee BO, Fan JY. Trajectories and associated factors of quality of life, global outcome, and post-concussion symptoms in the first year following mild traumatic brain injury. Qual Life Res. (2016) 25:2009-19. doi: 10.1007/s11136-015-1215-0

38. Cicerone KD, Kalmar K. Persistent postconcussion syndrome: the structure of subjective complaints after mild traumatic brain injury. J Head Trauma Rehabil. (1995) 10:1-17. doi: 10.1097/00001199-199510030-00002

39. Deb S, Lyons I, Koutzoukis C. Neuropsychiatric sequelae one year after a minor head injury. J Neurol Neurosurg Psychiatry. (1998) 65:899902. doi: 10.1136/jnnp.65.6.899

40. Emanuelson I, Andersson Holmkvist E, Björklund R, Stålhammar D. Quality of life and post-concussion symptoms in adults after mild traumatic brain injury: a population-based study in western Sweden. Acta Neurol Scand. (2003) 108:332-8. doi: 10.1034/j.1600-0404.2003. 00155.x

41. Fourtassi M, Hajjioui A, Ouahabi AE, Benmassaoud H, Hajjaj-Hassouni $\mathrm{N}$, Khamlichi AE. Long term outcome following mild traumatic brain injury in Moroccan patients. Clin Neurol Neurosurg. (2011) 113:71620. doi: 10.1016/j.clineuro.2011.07.010

42. Hanks RA, Temkin N, Machamer J, Dikmen SS. Emotional and behavioral adjustment after traumatic brain injury. Arch Phys Med Rehabil. (1999) 80:991-7. doi: 10.1016/S0003-9993(99)90049-7

43. Heitger MH, Jones RD, Frampton CM, Ardagh MW, Anderson TJ. Recovery in the first year after mild head injury: divergence of symptom status and self-perceived quality of life. Journal of Rehabil Med. (2007) 39:61221. doi: 10.2340/16501977-0100

44. Hellstrøm T, Westlye LT, Sigurdardottir S, Brunborg C, Soberg HL, Holthe $\varnothing$, et al. Longitudinal changes in brain morphology from 4 weeks to 12 months after mild traumatic brain injury: associations with cognitive functions and clinical variables. Brain Injury. (2017) 31:67485. doi: 10.1080/02699052.2017.1283537

45. Hsu HH, Lai WH, Yu HT, Xiao SH, Tsai YH, Wang KC, et al. Longterm presentation of postconcussion symptoms and associated factors: analysis of latent class modeling. Arch Clin Neuropsychol. (2021) 36:6273. doi: $10.1093 /$ arclin/acaa063

46. Krpan KM, Levine B, Stuss DT, Dawson DR. Executive function and coping at one-year post traumatic brain injury. J Clin Exp Neuropsychol. (2007) 29:36-46. doi: 10.1080/13803390500376816

47. Lecuyer Giguère F, Frasnelli A, De Guise É, Frasnelli J. Olfactory, cognitive and affective dysfunction assessed 24 hours and one year after a mild Traumatic Brain Injury (mTBI). Brain Injury. (2019) 33:118493. doi: 10.1080/02699052.2019.1631486

48. Lucas S, Smith BM, Temkin N, Bell KR, Dikmen S, Hoffman JM. Comorbidity of headache and depression after mild traumatic brain injury. Headache. (2016) 56:323-30. doi: 10.1111/head.12762

49. Losoi H, Silverberg ND, Wäljas M, Turunen S, Rosti-Otajärvi E, Helminen M, et al. Recovery from mild traumatic brain injury in previously healthy adults. J Neurotrauma. (2016) 33:766-76. doi: 10.1089/neu.2015.4070

50. McMahon P, Hricik A, Yue JK, Puccio AM, Inoue T, Lingsma HF, et al. Symptomatology and functional outcome in mild traumatic brain injury: results from the prospective TRACK-TBI study. J Neurotrauma. (2014) 31:2633. doi: $10.1089 /$ neu. 2013.2984

51. Oldenburg C, Lundin A, Edman G, Deboussard CN, Bartfai A. Emotional reserve and prolonged post-concussive symptoms and disability: a Swedish prospective 1-year mild traumatic brain injury cohort study. BMJ Open. (2018) 8:e020884. doi: 10.1136/bmjopen-2017-020884

52. Røe C, Sveen U, Alvsåker K, Bautz-Holter E. Post-concussion symptoms after mild traumatic brain injury: influence of demographic factors and injury severity in a 1-year cohort study. Disabil Rehabil. (2009) 31:123543. doi: 10.1080/09638280802532720

53. Sigurdardottir S, Andelic N, Roe C, Jerstad T, Schanke A. Postconcussion symptoms after traumatic brain injury at 3 and 12 months post-injury: a prospective study. Brain Injury. (2009) 23:489-97. doi: 10.1080/02699050902926309 
54. Skilbeck C, Dean T, Thomas M, Slatyer M. Impaired National Adult Reading Test (NART) performance in traumatic brain injury. Neuropsychol Rehabil. (2013) 23:234-55. doi: 10.1080/09602011.2012.747968

55. Steward KA, Gerstenecker A, Triebel KL, Kennedy R, Novack TA, Dreer LE, et al. Twelve-month recovery of medical decision-making capacity following traumatic brain injury. Neurology. (2016) 87:10529. doi: 10.1212/WNL.0000000000003079

56. Singh R, Choudhri K, Sinha S, Mason S, Lecky F, Dawson J. Global outcome after traumatic brain injury in a prospective cohort. Clin Neurol Neurosurg. (2019) 186:105526. doi: 10.1016/j.clineuro.2019.105526

57. Sterr A, Herron KA, Hayward C, Montaldi D. Are mild head injuries as mild as we think? neurobehavioral concomitants of chronic post-concussion syndrome. BMC Neurol. (2006) 6:7. doi: 10.1186/1471-2377-6-7

58. Teasdale G, Jennett B. Assessment of coma and impaired consciousness: a practical scale. The Lancet. (1974) 304:814. doi: 10.1016/S0140-6736(74)91639-0

59. Theadom A, Starkey NJ, Dowell T, Hume PA, Kahan M, McPherson K, et al. Sports-related brain injury in the general population: an epidemiological study. J Sci Med Sport. (2014) 17:591-6. doi: 10.1016/j.jsams.2014.02.001

60. Sport concussion assessment tool - 5th edition. Br J Sports Med. (2017) 51:851-8.

61. Greco T, Ferguson L, Giza C, Prins ML. Mechanisms underlying vulnerabilities after repeat mild traumatic brain injuries. Exp Neurol. (2019) 317:206-13. doi: 10.1016/j.expneurol.2019.01.012

62. Guskiewicz KM, McCrea M, Marshall SW, Cantu RC, Randolph C, Barr W, et al. Cumulative effects associated with recurrent concussion in collegiate football players: the NCAA concussion study. JAMA. (2003) 290:254955. doi: 10.1001/jama.290.19.2549

63. Master CL, Katz BP, Arbogast KB, McCrea MA, McAllister TW, Pasquina PF, et al. Differences in sport-related concussion for female and male athletes in comparable collegiate sports: a study from the NCAA-DoD Concussion Assessment, Research and Education (CARE) Consortium. Br J Sports Med. (2021) 55:1387-94. doi: 10.1136/bjsports-2020-103316

Conflict of Interest: The authors declare that the research was conducted in the absence of any commercial or financial relationships that could be construed as a potential conflict of interest.

Publisher's Note: All claims expressed in this article are solely those of the authors and do not necessarily represent those of their affiliated organizations, or those of the publisher, the editors and the reviewers. Any product that may be evaluated in this article, or claim that may be made by its manufacturer, is not guaranteed or endorsed by the publisher.

Copyright $\odot 2022$ Brady, Hume, Mahon and Theadom. This is an open-access article distributed under the terms of the Creative Commons Attribution License (CC BY). The use, distribution or reproduction in other forums is permitted, provided the original author(s) and the copyright owner(s) are credited and that the original publication in this journal is cited, in accordance with accepted academic practice. No use, distribution or reproduction is permitted which does not comply with these terms. 